\title{
A Tumor-Immune Interaction Model for Hepatocellular Carcinoma based on measured Lymphocyte Counts in Patients undergoing Radiotherapy
}

\author{
Wonmo Sung ${ }^{\mathrm{a}}$, Clemens Grassberger ${ }^{\mathrm{a}}$, Aimee Louise McNamara ${ }^{\mathrm{a}}$, Lucas Basler ${ }^{\mathrm{b}}$, Stefanie \\ Ehrbar $^{c}$, Stephanie Tanadini-Lang ${ }^{c}$, Theodore S. Hong ${ }^{\mathrm{a}}$, Harald Paganettia
}

aDepartment of Radiation Oncology, Massachusetts General Hospital/Harvard Medical School; bDepartment of Radiation Oncology, Paul Scherrer Institut, Villigen, Switzerland; 'Department of Radiation Oncology, University Hospital Zurich, Zurich, Switzerland;

\begin{abstract}
Purpose: The impact of radiation therapy on the immune system has recently gained attention particularly when delivered in combination with immunotherapy. However, it is unclear how different treatment fractionation regimens influence the interaction between the immune system and radiation. The goal of this work was to develop a mathematical model that quantifies both the immune stimulating as well as the immunosuppressive effects of radiotherapy and simulates the effects of different fractionation regimens based on patient data.
\end{abstract}

Methods and Materials: The framework describes the temporal evolution of tumor cells, lymphocytes, and inactivated dying tumor cells releasing antigens during radiation therapy, specifically modeling how recruited lymphocytes inhibit tumor progression. The parameters of the model were partly taken from the literature and in part extracted from blood samples (circulating lymphocytes: CLs) collected from hepatocellular carcinoma patients undergoing radiotherapy and their outcomes. The dose volume histograms to circulating lymphocytes were calculated with a probability-based model.

Results: Based on the fitted parameters, the model enabled a study into the depletion and recovery of CLs in patients as a function of fractionation regimen. Our results quantify the ability of short fractionation regimens to lead to shorter periods of lymphocyte depletion and predict faster recovery after the end of treatment. The model shows that treatment breaks between fractions can prolong the period of lymphocyte depletion and should be avoided.

Corresponding author: Harald Paganetti, PhD, Department of Radiation Oncology, Massachusetts General Hospital, 100 Blossom Street, Boston, MA, 02114, Tel: 617-726-5847; hpaganetti@mgh.harvard.edu.

Publisher's Disclaimer: This is a PDF file of an unedited manuscript that has been accepted for publication. As a service to our customers we are providing this early version of the manuscript. The manuscript will undergo copyediting, typesetting, and review of the resulting proof before it is published in its final form. Please note that during the production process errors may be discovered which could affect the content, and all legal disclaimers that apply to the journal pertain.

Conflict of interest: None 
Conclusions: This study introduces a mathematical model for tumor-immune interactions using clinically extracted radiotherapy patient data, which can be applied to design trials aimed at minimizing lymphocyte depleting effects in radiation therapy.

\section{INTRODUCTION}

Radiotherapy is widely used as a cancer treatment either alone or in combination with systemic or targeted therapies [1-3]. In addition to killing tumor cells via radiation-induced DNA damage, radiation can also enhance tumor immunogenicity through various mechanisms [4,5], among them the release of tumor-associated antigens and dangerassociated molecular patterns [6,7]. Additionally, radiation can also cause rapid depletion of circulating lymphocytes (CLs) [8]. Thus, radiation has both immunostimulant and immunosuppressive effects. Current computational efforts for treatment planning focus on the effect of radiation protocols on tumor control and toxicity [9], but not on the impact on the immune response. Growing evidence has demonstrated that lymphocyte counts are correlated with patient response to immune checkpoint inhibitors (ICIs) [10,11]. It is hypothesized that radiotherapy patients with less severe lymphopenia are in a better position to derive benefit from combination therapy with ICIs. Therefore, a proper understanding of the interaction between tumor and immune cells is essential to aid the design of immunogenic RT treatment strategies that are not only efficient but also free of adverse side effects [12].

Liver cancer is the fifth most common cancer among men in the world [13]. Despite improvements in treatment strategies, the 5-year survival rate of affected liver patients remains below $15 \%$ [14] and the intra- and extra-hepatic metastasis rate remains high after treatment [15]. Radiation therapy (RT) as a treatment option induces not only direct tumor cell kill but also phenotype alteration of tumor cells, which may affect tumor immunogenicity [16,17]. Tumor antigen and pro-inflammatory cytokines are released, leading to recruitment of dendritic cells and the stimulation of an antigen-specific $\mathrm{T}$ cell response[18]. Immune biomarkers in the blood are correlated with overall survival after radiotherapy, suggesting radiation may play a role in triggering an immune response in liver patients [19]. Furthermore, since CLs are known to be highly radiosensitive [20], the quantification of radiation-damaged CLs is important to balance these effects. Recently, probability-based approaches have been developed to calculate the radiation dose to the CLs for both brain and liver cancer patients [21,22].

In this study, we propose a bio-mathematical model to describe the interaction of hepatocellular carcinoma (HCC) and the immune system in patients treated with radiotherapy, based on measured lymphocyte counts during treatment. Previous models were developed mainly for preclinical research and were focused on animal models[23-25]. To our knowledge, the present study is the first approach to formalize a bio-mathematical model for tumor-immune interactions using clinically extracted radiotherapy patient data, i.e. based on blood samples taken during and after treatment. We conceptually follow an approach previously established for animal models and based on ad-hoc estimates of model parameters $[23,26,27]$. 


\section{METHODS AND MATERIALS}

\section{Patient cohort}

The study is based on data from 17 HCC patients treated at our institution between 2011 and 2014 as part of a clinical trial. All patients were treated with passively scattered proton therapy without chemotherapy receiving on average 15 fractions to a total dose of $58.0 \mathrm{~Gy}$ (RBE) (median) with a relative biological effectiveness (RBE) of 1.1 according to institutional guidelines. The median volume of the gross target volume (GTV) and whole liver were 107.1 and $1590.8 \mathrm{~cm}^{3}$, respectively. CL counts were collected at day 1 before (baseline), during (day 8-20), and after treatment (after day 21). The detailed patient characteristics are provided in supplementary table 1 and have been published previously $[19,28]$.

\section{Model equations and assumptions}

We describe the dynamics of the tumor and immune cell populations using a system of ordinary differential equations (ODEs) adapted from the predator-prey model $[23,26,27]$ and outlined in equations $(\mathrm{a}-\mathrm{d})$ and in figure 1 .

$T, I, M$, and $L$ denote the number of primary tumor cells, inactivated tumor cells releasing antigens, metastatic (i.e. non-local) tumor cells, and circulating lymphocytes, respectively. The primary tumor cell compartment can be described as

$$
\frac{d T}{d t}=\overbrace{a T}^{\text {tumor growth }} \overbrace{-\omega_{1} \frac{\mathrm{T}}{g+T+M} L-\delta^{*}\left(t_{R}\right)\left(1-e^{\left.-\alpha_{T} D_{T}-\beta_{T} D_{T}^{2}\right)} T\right.}^{\text {radiation kill }}
$$

We assume that the undisturbed tumor grows exponentially with a growth rate of $a$, and that its growth is suppressed by the immune system. In order to account for a saturation effect of the immune response in the clearance term, we use a Monod form $(T /(g+T))$ (also called Michaelis-Menten term) with a half-saturation constant $g$. This form accounts for a reduced portion of increasing tumor volume to be in contact with circulating lymphocytes as described by Kirschner and Panetta [29]. The linear quadratic (LQ) model is used to describe cell survival after each treatment fraction using $a_{T}$ and $\beta_{T}$ as parameters for the intrinsic radiosensitivity of tumor cells and $D_{T}$ as the dose homogenously delivered to the cells at each fraction. The $\delta *\left(\mathrm{~T}_{\mathrm{R}}\right)$ term is the Dirac delta function restricting the effect of instantaneous radiation to a specific treatment time point $t_{R}$. (see equations (e)- $(\mathrm{g})$ for more descriptions).

The non-clonogenic, inactivated tumor cells (I) that are irradiated cells stimulating the immune response, decay exponentially $(-r I)$ :

$$
\frac{d I}{d t}=\overbrace{\delta^{*}\left(t_{R}\right)\left(1-e^{-\alpha_{T} D_{T}-\beta_{T} D_{T}^{2}}\right) T \stackrel{\text { radiation kill }}{\text { decay }}}^{\text {rerI }}
$$


The metastatic tumor cells $(M)$, defined as the non-local and non-irradiated population, are assumed to have the same growth and inhibition rates as the primary tumor cells. Due to their distance to the main target we assume no radiation effects.

$$
\frac{d M}{d t}=\overbrace{a M}^{\text {tumorgrowth }} \overbrace{-\omega_{1} \frac{M}{g+T+M} L}^{\text {immune }- \text { induced death }}
$$

Lastly, the dynamics of the circulating lymphocytes are described by

$$
\begin{aligned}
& \frac{d L}{d t}=\overbrace{\omega_{2} \frac{T+M}{g+T+M} L+\omega_{3} \frac{I}{g+I} L}^{\text {recruitment }} \underbrace{+s}_{\text {supply }}
\end{aligned}
$$

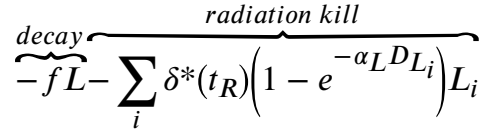

The first two terms describe the lymphocyte recruitment due to antigen release by the primary and metastatic tumor $(T+M)$ and the inactivated tumor cells $(I)$. Assuming similar saturation dynamics, the same $\mathrm{g}$ factor is used for all tumor-immune interaction terms. However, immune activation is different for clonogenic tumor cells and those inactivated by irradiation as parameterized by $\omega_{2}$ and $\omega_{3}$, respectively. This separation is intended to separate the existing, insufficient lymphocyte activation from the additional activation due to the specific antigen release caused by radiation. The lymphocyte population is assumed to increase due to supply from other compartments, such as regenerative activity in the bone marrow $(+s)$ and at the same time decay exponentially as a function of time $(-f L)$. For immune cells, the linear survival model was used to describe cell kill with a radiation parameter for CLs $\left(a_{L}\right)$. For the dose-response relationships, $D_{L_{i}}$ is the dose per fraction delivered to a given volume of CLs $\left(L_{i}\right.$ and $\left.\sum_{i} L_{i}=L\right)$ which is provided by a dose volume histogram (DVH) for the circulating blood as a surrogate for the circulating lymphocytes. The DVH was calculated with a probability-based model [22] (Supplementary Material 1), which calculates the fraction of lymphocytes receiving different dose levels based on dose and blood flow to each liver compartment.

Instead of solving differential equations (a)-(d) directly with $\delta^{*}\left(T_{R}\right)$ terms, radiation cell kill is separately described by instantaneous changes in $\mathrm{T}$ and $\mathrm{I}$ at discrete timepoints, i.e. the change over a single fixed time step, $\mathrm{n}$ to $\mathrm{n}+1$, is expressed as

$$
\begin{gathered}
T_{n+1}=T_{n} \cdot e^{-\alpha_{T} D_{T}-\beta_{T} D_{T}^{2}} \\
I_{n+1}=I_{n}+T_{n} \cdot\left(1-e^{-\alpha_{T} D_{T}-\beta_{T} D_{T}^{2}}\right)
\end{gathered}
$$




$$
L_{n+1}=\sum_{i} L_{n i} \cdot e^{-\alpha L D_{i}}
$$

The ODEs described above (a-d) excluding the $\delta^{*}\left(T_{R}\right)$ terms are then used to model the behavior of the tumor-immune system between fractions.

\section{Parameters}

Our model based on equations (a)-(d) has a total of eleven parameters, listed in Table 1. Four of those parameters were fixed according to published values. Seven parameters were fitted to patient CL counts obtained during and after radiotherapy and the patterns of failure.

a) Parameters taken from the literature-For the parameters describing the clonogenic tumor cells (equation (a) and (c)) we use an exponential tumor growth model in the absence of immune response. We chose the exponential form not only to minimize the number of parameters but also to separate the tumor suppressant term caused by lymphocytes. Other tumor growth models such as the Gompertz formulation intrinsically include decelerating growth effects from, for instance, immune surveillance. The human tumor volume doubling time without involvement of the immune system was assumed to be 88 days $\left(a=0.01\right.$ days $\left.^{-1}\right)$, which was the maximum tumor growth rate reported for HCC patients[30]. It is important to use parameters for human tumor growth, as growth rates in nude mice have been reported to be several times higher $\left(0.05\right.$ days $\left.^{-1}\right)$. [31]

For the parameters of equation (b), describing the non-clonogenic inactivated tumor cells, the same radiation effect parameters were used. The inactivated tumor cells were assumed to decay exponentially with a half-life of 5 days $\left(r=0.14\right.$ days $\left.^{-1}\right)$. [32]

For the parameters in the lymphocyte equation (d), the lifetime of lymphocytes was assumed to be 30 days, i.e. $f=0.033$ days $^{-1}$. [33]

b) Parameters extracted from clinical data-The clinical data of CL levels in blood samples and the patterns of failure were used to fit the tumor-immune interaction parameters $\left(\omega_{1}, \omega_{2}, \omega_{3}, g\right)$, lymphocyte supply $(s)$, and radiosensitivity $\left(\alpha_{T}, \beta_{T}\right.$, and $\left.\alpha_{L}\right)$ of tumor and lymphocytes, which are considered to be disease-specific. Their parameter sensitivity was analyzed (supplementary figure 2).

We extracted the tumor-immune interaction parameters by fitting the model to clinical data of CL levels in blood samples taken during and after radiotherapy. The three interaction terms were constrained to the interval $\omega_{2}, \omega_{3} \in\left[10^{-3}, 10^{\circ}\right]$ days $^{-1}$ [23]. We assumed saturation parameters within $g \in\left[10^{8}, 10^{14}\right]$. The initial radiation effect parameters to guide the fit were chosen based on clinical HCC data $\left(a_{T}=0,037 G^{-1}\right.$ and $\left.a_{T} / \beta_{T^{=}}=14.3 \mathrm{~Gy}\right)$ [34]. A single radiosensitivity parameter $\left(a_{L}\right)$ was assumed to describe the linear slopes of the lymphocyte dose response curve because they are extremely radio-sensitive cells and do not show a shoulder in measured survival curves. This is not a pure cell survival parameter because the model is sensitive to CL kill and inactivation so that $a_{\mathrm{L}}$ covers both. The fitting model was implemented in Python 3.7 with the lmfit package using Powell's method. 
Additionally the immune-induced death rate $\left(\omega_{1}\right)$ and tumor radiosensitivity $\left(\mathrm{a}_{\mathrm{L}}\right)$ were fitted to acquire 5\% local failure and distant metastasis rate $40 \%$ at two years [28] for a 15 -fraction treatment with standard deviation of the normal distribution tumor radiosensitivity $\left(\sigma_{\alpha_{T}}=0.006\right)$ [34], as was observed in our clinical data for this patient cohort. Instead of using lmfit package, grid search was used to find these parameters (Figure 2).

Confidence intervals were estimated using bootstrap samples to test the robustness and possible ranges of the derived parameter values. Samples were randomly selected with replacement to create 1000 different datasets, yielding a distribution of possible parameter values from which confidence intervals were calculated.

\section{Baseline values}

We estimated the baseline number of HCC tumor cells as $1.07 \times 10^{11}$ derived by a tumor cell density of $10^{9}$ per $\mathrm{cm}^{3}$ [35] and a median GTV volume of $107 \mathrm{~cm}^{3}$ in our cohort. Considering a conservative detection capability for current diagnostic technology $(1 \mathrm{cc}=$ $\left.10^{9}\right)$, the number of metastatic tumor cells is assumed to be $0.1 \%\left(1.07 \times 10^{8}\right)$ proportional to the primary tumor volume, as further discussed below. The average baseline number of circulating lymphocytes was determined by multiplying the average lymphocyte counts $\left(1122 \pm 469\right.$ per $\left.\mathrm{mm}^{3}\right)$ in our cohort with the average total body blood volume $(5 \mathrm{~L})$ resulting in $5.61 \times 10^{9}$ lymphocytes.

\section{Simulations}

The final model is able to simulate trajectories of CLs for different radiation fractionation regimens. We applied eleven different fractionation regimens ( 3 to 10, 15, 20, and 25 fractions) and four different time intervals between fractions (1, 2, 3, 4 days). All treatment schedules have an identical biological effective dose (BED) of $73.8 \mathrm{~Gy}$, which is equivalent to a total dose of $58 \mathrm{~Gy}(\mathrm{RBE})$ delivered in 15 fractions.

\section{RESULTS}

For 17 HCC patients, we collected 118 absolute lymphocyte counts (ALC, on average 7 counts for each patient and range 3-12 counts per patient) normalized to the value of each patient's baseline. All of the patients experienced lymphocyte depletion during radiotherapy (Figure 3(a)). Similar patterns of immunosuppression and recovery have been reported by Gustafson and Byun for liver cancer patients treated with RT [36,37]. The tumor-immune cell interaction parameters $\left(\omega_{1}, \omega_{2}, \omega_{3}, g\right)$, lymphocyte supply $(s)$, and radiosensitivity $\left(a_{T}\right.$, $\beta_{T}$, and $a_{L}$ ) of tumor and lymphocytes were fit to minimize the differences between the data and model solutions (Figure 3(b)). Figure 3(b) also shows the 95\% confidence intervals with the parameters calculated using 1000 bootstrap samples. The difference between the circulating lymphocyte trajectories before (f1) and after (f2) the grid search was negligible. The chosen best fit values for the tumor - immune parameters are listed with 95\% confidence intervals in table 1 . The robustness of those parameters was assessed by applying a Chi-Square goodness of fit test and ranges of fixed parameters (Supplementary Figure 3 and Table 2). This two-step method significantly improved the goodness of fit to local 
failure / distant metastasis while maintaining the one to lymphocyte counts and demonstrated the robustness of the obtained parameter values.

By using the parameters described in the Methods section, the model simulates not only the population of immune cells but also the tumor and inactivated tumor cells as a function of time (Figure 3(b)). Radiation initially reduces the clonogenic tumor cell count during therapy, while generating inactivated tumor cells that continue to release antigens. These inactivated tumor cells eventually decay. The fitted parameters for lymphocyte recruitment by inactivated tumor cells $\left(\omega_{3}\right)$ was higher than the one by clonogenic tumor cells $\left(\omega_{2}\right)$, which is consistent with the hypothesis of antigens generated by inactivated tumor cells being central to mount an effective tumor-directed immune response. The remaining clonogenic tumor cells after radiotherapy may be eliminated by CLs recovering to their baseline number.

We applied the model to simulate trajectories of CLs for different radiation fractionation regimens. Figure 4 shows examples of simulated CL populations for different fractionation regimens, delivered daily on weekdays. As expected, the number of inactivated tumor cells were maximized when a smaller number of fractions was used. Hypofractionation causes not only a longer lymphocyte depletion period but also maximizes the number of inactivated tumor cells in a shorter period. (Figure 4(a)). Lymphocyte counts eventually recover, but shorter regimens also lead to an expedited recovery because of the larger inactivated tumor cell population that stimulates lymphocytes: the model predicts on average only 35 days for lymphocytes to recover to $70 \%$ of their baseline values after 3 fractions, in contrast to 54 days after 15 fractions. This is qualitatively consistent with clinical observations that patients treated with more fractions had severe lymphopenia at one month after starting radiation [8]. Also, the experience from previous pre-clinical studies suggest that the immune effects of radiation are generally higher when using hypofractionation $[7,38]$. The number of inactivated tumor cells, which are vital for the initiation of an early immune response in radiotherapy, was maximized on the first- and third- day during treatment with 3 and 15 fractions, respectively. Treatment intervals also affect the trajectories of CLs over the course of a treatment (Figure 4(b)). For 15 fractions, single daily delivery takes on average 54 days to recover to $70 \%$ of their baseline. On the other hand, hypothetical schedules treating only 2 days per week would take 77 days to recover. Our model clearly indicates that hypofractionation is far more effective than conventional fractionation when the aim is to increase the number of lymphocytes in circulation during and shortly after RT (see Figure 4 a).

We subsequently applied the model to study the effect of different fractionation regimens on lymphocyte recovery. The model predicts substantial changes in recovery time with different fractionation regimens (Figure 5) while distant metastasis rates remained stable (Supplementary Figure 4). The difference of recovery time between 3 and 25 daily fractions could be up to a month after radiotherapy. Longer treatment breaks have a detrimental effect on the lymphocyte recovery time post treatment, especially for longer fractionation schemes. Interestingly, the ratio of the initial number of tumor cells to lymphocytes is related to tumor control at 2 years, though the ratio has to be changed substantially to measure progressive disease (Supplementary Figure 5). 


\section{DISCUSSION}

We developed a model to describe the effects of the circulating lymphocytes on hepatocellular carcinoma growth treated with radiation. For the first time, an immune-tumor interaction model is based on parameters derived from patient blood counts taken before, during and after radiotherapy. Previous models that aim to simulate the tumor-immune interaction have been based exclusively on animal data [23-27]. The framework allows the prediction of lymphocyte counts during fractionated radiotherapy treatments and can be used to help design and optimize clinical trials that aim at enhancing tumor immunogenicity using radiation.

The underlying motivation for the specific structure of our model are clinical observations that fractionation [39], irradiation volume[11] and total dose delivered impact the circulating lymphocyte count. Rather than predicting absolute results for individual patients, the purpose of this work is to fit population averaged parameters to the model, enabling us to study the immunologic consequences of different radiotherapy regimen for representative populations. We assumed the initial fraction of metastasis to be $0.1 \%$ and defined recurrence as this compartment reaching $1 \mathrm{cc}$ in volume, i.e. detectable by PET. Our results are robust towards this assumption, as changing this threshold only affects $\omega_{1}$, as for the primary compartment the majority of the cell kill stems from RT. Therefore, choosing a different initial size for the metastatic compartment leads to a model that behaves similarly with a slightly adjusted $\omega_{1}$ (see supplementary figure 2). The current model considers lymphocyte dynamics during RT only, and is an essential step on the way to more advanced models that will include the effects of immune checkpoint inhibitors in combination with RT.

One of the critical inputs is the dose to the blood pool and the circulating lymphocytes. The current liver blood DVH model uses a probability weighting factor with re-circulation and occupation times of the blood in segments of the liver [22]. As a consequence, the model is only applicable if the beam on-time is shorter than the total blood circulation time $(200 \mathrm{sec})$ and longer than the blood occupation time in the organ of interest (23 sec) (equation (j) and (k) in the Supplementary Material 1). Consequently, we did set the minimum and maximum fraction sizes to 3 and 25, respectively. Moreover, 3 fractions are currently the lowest number of fractions used for liver treatments. To extend the model further, e.g. to single dose delivery, would require more careful consideration of normal tissue toxicities [40].

Currently, the immune system is not considered an organ at risk (OAR) in treatment planning despite reported associations of lymphopenia with inferior outcomes $[8,18,37]$. The concept of seeing the immune system as an OAR will become more important in the context of combined radiotherapy-immunotherapy [5], not only for HCC patients but also for other indications in which such combination regimens are explored [41]. For other treatment sites, the effect of radiation on the lymphocyte compartment might be very different due to the underlying structure of lymph nodes and blood vessels $[42,43]$.

Our study is based on a single-institution cohort treated with proton therapy. Others have reported that lymphopenia occurs after HCC treatments using conventional, photon based RT $[37,44]$. The limited beam range inherent to proton therapy eliminates the low-dose 
region distal to the target [45], while the "low-dose bath" from photons may introduce more radiation dose to the circulating blood and lymph nodes. The longer beam-on time using photon RT is also likely to damage a higher number of circulating lymphocytes. We also observed that longer overall treatment time, i.e. with RT fractions given every second day, reduced the amount of lymphocyte depletion (see Figure 4). Not only does this reduce severity of the nadir, it also boosts the ALC during the first two weeks by up to 50\%. Our model used proton-specific dose to the blood as an input, however it could similarly use dose based on photon treatment plans, which would lead to more lymphocyte depletion as observed in clinical data $[46,47]$.

Recently, Alfonso et al. studied the optimal sequencing of RT and surgery in the context of RT induced anti-tumor immunity [48]. Our study differs as it only considers inoperable tumors, where radiation and systemic therapy are the only option. Additionally, our initial tumor cell numbers are based on actual tumor sizes, and are significantly higher, which leads to large differences in dependent parameters such as the saturation constant and lysis rate [49].

The model is motivated by the fact that we can measure the circulating compartment during therapy, and because new tumor-infiltrating lymphocytes are constantly supplied by other lymphocyte compartments, mainly via the circulating blood, i.e. they do correlate [50]. Other approaches, for example Serre et al [25], formulate a component of immune effector cells at the tumor site which, while possibly a more relevant quantity, cannot be practically observed at different time point during treatment. Due to the strong correlations observed between outcome and peripheral lymphocyte counts and the availability of measured patient data at multiple time points, we opted to include the peripheral lymphocytes as compartment to apply the model in a clinical context.

We separated the lymphocyte recruitment by considering existing tumor burden and radiotherapy-induced stimulation separately, i.e. $\mathrm{w} 2 \neq \mathrm{a} 3$. Clinical and preclinical studies have shown that radiation modulates the immune environment, which is different from the baseline antigen environment caused by the clonogenic tumor burden [51]. This radiationinduced stimulation might be modulated by, for example, additional antigen presentation or $\mathrm{T}$ cell infiltration driven by inflammation and is therefore described by a separate term (w3) Sotolongo et al. [27] used w2.T.L + w3-I.L to classify lymphocyte stimulation. Along similar lines, Alfonso et al. [48] considered different parameters in a lymphocyte equation for tumor burden and tumor cell sterilized by radiotherapy as w2.T/(T+g).L + w3.I, and Serre et al. [25] formalized them in a similar manner. We used an empirical Monod equation to describe the saturation effect of the immune response and as this functional form is motivated by geometrical assumptions [ref], we used the same g parameter for the terms driven by clonogenic $(\mathrm{T})$ and inactivated tumor $(\mathrm{I})$.

We do not describe the tumor-immune interaction including multiple cell populations, such as dendritic and natural killer cells [52]. Furthermore, we do not include oligoclonality of $\mathrm{T}$ cells, i.e. the fact that a lower nadir of $\mathrm{T}$ cells at the end of therapy could lead to an expansion of a limited number of sub-clones that is not effective in containing a heterogenous tumor cell population [53]. Additional information about patient-specific 
immune responses and subtypes of cells during and after RT could allow for more specific models albeit requiring more parameters [5].

The main goal and a unique feature of our approach is that a minimum number of model parameters are derived from clinical observables (i.e. blood counts, tumor size, observed outcomes), while preserving some mechanistic features such as the antigen release by inactivated tumor cells and the differential effects of radiation on tumor cells and lymphocytes. The model is able to quantitatively describe how shorter treatment regimen lead to less radiation-induced lymphopenia and can reduce immune recovery periods after RT, which can lead to months of additional immunosuppression. While we demonstrate that model parameters can be extracted from patient data and fit average population dynamics, our model predictions need to be validated using cohorts treated with different fractionation regimen and treatment modalities. Furthermore, future work needs to investigate the ability of the model to help in the prediction of individual patient outcomes, and include the effect of other treatment modalities, particularly immunotherapy.

\section{Supplementary Material}

Refer to Web version on PubMed Central for supplementary material.

\section{Funding statement:}

This work was funded by the National Institutes of Health through R21 CA241918 (PI: C. Grassberger).

\section{REFERENCES}

[1]. Grassberger C, G Scott J, Paganetti H. Biomathematical Optimization of Radiation Therapy in the Era of Targeted Agents. Int J Radiat Oncol Biol Phys 2017;97:13-7. doi:10.1016/ j.ijrobp.2016.09.008. [PubMed: 27979444]

[2]. Geng C, Paganetti H, Grassberger C. Prediction of Treatment Response for Combined Chemo- and Radiation Therapy for Non-Small Cell Lung Cancer Patients Using a Bio-Mathematical Model. Sci Rep 2017:1-12. doi:10.1038/s41598-017-13646-z. [PubMed: 28127051]

[3]. Grassberger C, McClatchy DM, Geng C, Kamran SC, Fintelmann F, Maruvka YE, et al. Patientspecific tumor growth trajectories determine persistent and resistant cancer cell populations during treatment with targeted therapies. Cancer Res 2019:epub. doi:10.1158/0008-5472.CAN-18-3652.

[4]. Demaria S, Golden EB, Formenti SC. Role of Local Radiation Therapy in Cancer Immunotherapy. JAMA Oncol 2015;1:1325-32. doi:10.1001/jamaoncol.2015.2756. [PubMed: 26270858]

[5]. Grassberger C, Ellsworth SG, Wilks MQ, Keane FK, Loeffler JS. Assessing the interactions between radiotherapy and antitumour immunity. Nat Rev Clin Oncol 2019;11:805. doi:10.1038/ s41571-019-0238-9.

[6]. Ciernik IF, Romero P, Berzofsky JA, Carbone DP. Ionizing radiation enhances immunogenicity of cells expressing a tumor-specific T-cell epitope. Int J Radiat Oncol Biol Phys 1999;45:735-41. doi:10.1016/S0360-3016(99)00226-6. [PubMed: 10524430]

[7]. Vanpouille-Box C, Alard A, Aryankalayil MJ, Sarfraz Y, Diamond JM, Schneider RJ, et al. DNA exonuclease Trex 1 regulates radiotherapy-induced tumour immunogenicity. Nat Commun 2017;8:15618. doi:10.1038/ncomms15618. [PubMed: 28598415]

[8]. Wild AT, Herman JM, Dholakia AS, Moningi S, Lu Y, Rosati LM, et al. Lymphocyte-Sparing Effect of Stereotactic Body Radiation Therapy in Patients With Unresectable Pancreatic Cancer. Int J Radiat Oncol Biol Phys 2016;94:571-9. doi:10.1016/j.ijrobp.2015.11.026. [PubMed: 26867885] 
[9]. Ajdari A, Niyazi M, Nicolay NH, Thieke C, Jeraj R, Bortfeld T. Towards optimal stopping in radiation therapy. Radiother Oncol 2019;134:96-100. doi:10.1016/j.radonc.2019.01.010. [PubMed: 31005230]

[10]. Martens A, Wistuba-Hamprecht K, Foppen MG, Yuan J, Postow MA, Wong P, et al. Baseline Peripheral Blood Biomarkers Associated with Clinical Outcome of Advanced Melanoma Patients Treated with Ipilimumab. Clin Cancer Res 2016;22:2908-18. doi:10.1158/1078-0432.CCR-15-2412. [PubMed: 26787752]

[11]. Ellsworth SG. Field size effects on the risk and severity of treatment-induced lymphopenia in patients undergoing radiation therapy for solid tumors. Adv Radiat Oncol 2018;3:512-9. doi:10.1016/j.adro.2018.08.014. [PubMed: 30370350]

[12]. Dewan MZ, Galloway AE, Kawashima N, Dewyngaert JK, Babb JS, Formenti SC, et al. Fractionated but Not Single-Dose Radiotherapy Induces an Immune-Mediated Abscopal Effect when Combined with Anti-CTLA-4 Antibody. Clin Cancer Res 2009;15:5379-88. doi:10.1158/1078-0432.CCR-09-0265. [PubMed: 19706802]

[13]. Stewart BW, Kleihues P. World cancer report. 2003.

[14]. Momin BR, Pinheiro PS, Carreira H, Li C, Weir HK. Liver cancer survival in the United States by race and stage (2001-2009): Findings from the CONCORD-2 study. Cancer 2017;123:505978. doi:10.1002/cncr.30820. [PubMed: 29205306]

[15]. Uchino K, Tateishi R, Shiina S, Kanda M, Masuzaki R, Kondo Y, et al. Hepatocellular carcinoma with extrahepatic metastasis. Cancer 2011;117:4475-83. doi:10.1002/cncr.25960. [PubMed: 21437884]

[16]. Garnett CT, Palena C, Chakarborty M, Tsang K-Y, Schlom J, Hodge JW. Sublethal Irradiation of Human Tumor Cells Modulates Phenotype Resulting in Enhanced Killing by Cytotoxic T Lymphocytes. Cancer Res 2004;64:7985-94. doi:10.1158/0008-5472.CAN-04-1525. [PubMed: 15520206]

[17]. Makinde AY, John-Aryankalayil M, Palayoor ST, Cerna D, Coleman CN. Radiation Survivors: Understanding and Exploiting the Phenotype following Fractionated Radiation Therapy. Mol Cancer Res 2013;11:5-12. doi:10.1158/1541-7786.MCR-12-0492. [PubMed: 23175523]

[18]. Popp I, Grosu AL, Niedermann G, Duda DG. Immune modulation by hypofractionated stereotactic radiation therapy: Therapeutic implications. Radiother Oncol 2016;120:185-94. doi:10.1016/j.radonc.2016.07.013. [PubMed: 27495145]

[19]. Grassberger C, S Hong T, Hato T, Y Yeap B, Y Wo J, Tracy M, et al. Differential Association Between Circulating Lymphocyte Populations With Outcome After Radiation Therapy in Subtypes of Liver Cancer. Int J Radiat Oncol Biol Phys 2018;101:1222-5. doi:10.1016/ j.ijrobp.2018.04.026. [PubMed: 29859792]

[20]. Nakamura N, Kusunoki Y, Akiyama M. Radiosensitivity of CD4 or CD8 Positive Human TLymphocytes by an in Vitro Colony Formation Assay. Radiat Res 2010;123:224.

[21]. Yovino S, Kleinberg L, Grossman SA, Narayanan M, Ford E. The Etiology of Treatment-related Lymphopenia in Patients with Malignant Gliomas: Modeling Radiation Dose to Circulating Lymphocytes Explains Clinical Observations and Suggests Methods of Modifying the Impact of Radiation on Immune Cells. Cancer Invest 2013;31:140-4. doi:10.3109/07357907.2012.762780. [PubMed: 23362951]

[22]. Basler L, Andratschke N, Ehrbar S, Guckenberger M, Tanadini-Lang S. Modelling the immunosuppressive effect of liver SBRT by simulating the dose to circulating lymphocytes: an in-silico planning study. Radiat Oncol 2018:1-8. doi:10.1186/s13014-018-0952-y. [PubMed: 29304828]

[23]. Rodriguez-Perez D, Sotolongo-Grau O, Espinosa Riquelme R, Sotolongo-Costa O, Santos Miranda JA, Antoranz JC. Assessment of cancer immunotherapy outcome in terms of the immune response time features. Math Med Biol 2007;24:287-300. doi:10.1093/imammb/ dqm003. [PubMed: 17652107]

[24]. Poleszczuk J, Enderling H. The Optimal Radiation Dose to Induce Robust Systemic Anti-Tumor Immunity. Ijms 2018;19:3377. doi:10.3390/ijms 19113377. 
[25]. Serre R, Benzekry S, Padovani L, Meille C, Andre N, Ciccolini J, et al. Mathematical Modeling of Cancer Immunotherapy and Its Synergy with Radiotherapy. Cancer Res 2016;76:4931-40. doi:10.1158/0008-5472.CAN-15-3567. [PubMed: 27302167]

[26]. Sotolongo-Costa O, Morales Molina L, Rodriguez-Perez D, Antoranz JC, Chacón Reyes M. Behavior of tumors under nonstationary therapy. Physica D 2003;178:242-53. doi:10.1016/ S0167-2789(03)00005-8.

[27]. Sotolongo-Grau O, Rodriguez-Perez D, Santos Miranda JA, Sotolongo-Costa O, Antoranz JC. Immune system-tumour efficiency ratio as a new oncological index for radiotherapy treatment optimization. Math Med Biol 2009;26:297-307. doi:10.1093/imammb/dqp005. [PubMed: 19584118]

[28]. Hong TS, Wo JY, Yeap BY, Ben-Josef E, McDonnell EI, Blaszkowsky LS, et al. MultiInstitutional Phase II Study of High-Dose Hypofractionated Proton Beam Therapy in Patients With Localized, Unresectable Hepatocellular Carcinoma and Intrahepatic Cholangiocarcinoma. J Clin Oncol 2016;34:460-8. doi:10.1200/JCO.2015.64.2710. [PubMed: 26668346]

[29]. De Boer RJ, Hogeweg P, Dullens HF, De Weger RA, Otter Den W. Macrophage T lymphocyte interactions in the anti-tumor immune response: a mathematical model. The Journal of Immunology 1985;134:2748-58. [PubMed: 3156189]

[30]. Park Y, Choi D, Lim HK, Rhim H, Kim Y-S, Kim SH, et al. Growth Rate of New Hepatocellular Carcinoma After Percutaneous Radiofrequency Ablation: Evaluation with Multiphase CT. Am J Roentgenol 2012;191:215-20. doi:10.2214/AJR.07.3297.

[31]. Zhang R, Ma L, Zheng M, Ren J, Wang T, Meng Y, et al. Survivin knockdown by short hairpin RNA abrogates the growth of human hepatocellular carcinoma xenografts in nude mice. Cancer Gene Ther 2009;17:275-88. doi:10.1038/cgt.2009.68. [PubMed: 19876077]

[32]. Serre R, Barlesi F, Muracciole X, Barbolosi D. Immunologically effective dose: a practical model for immuno-radiotherapy. Oncotarget 2018;9:31812. doi:10.18632/oncotarget.25746. [PubMed: 30159124]

[33]. Kuznetsov VA, Makalkin IA, Taylor MA, Perelson AS. Nonlinear dynamics of immunogenic tumors: Parameter estimation and global bifurcation analysis. B Math Biol 1994;56:295-321. doi:10.1007/BF02460644.

[34]. Tai A, Erickson B, Khater KA, Li XA. Estimate of Radiobiologic Parameters From Clinical Data for Biologically Based Treatment Planning for Liver Irradiation. Int J Radiat Oncol Biol Phys 2008;70:900-7. doi:10.1016/j.ijrobp.2007.10.037. [PubMed: 18262101]

[35]. Devita VT, Young RC, Canellos GP. Combination versus single agent chemotherapy: A review of the basis for selection of drug treatment of cancer. Cancer 1975;35:98-110. doi:10.1002/1097-0142(197501)35:1<98::AID-CNCR2820350115>3.0.CO;2-B. [PubMed: 162854]

[36]. Gustafson MP, Bornschlegl S, Park SS, Gastineau DA, Roberts LR, Dietz AB, et al. Comprehensive assessment of circulating immune cell populations in response to stereotactic body radiation therapy in patients with liver cancer. Adv Radiat Oncol 2017;2:540-7. doi:10.1016/j.adro.2017.08.003. [PubMed: 29204520]

[37]. Byun HK, Kim N, Park S, Seong J. Acute severe lymphopenia by radiotherapy is associated with reduced overall survival in hepatocellular carcinoma. Strahlenther Onkol 2019;18:1-11. doi:10.1007/s00066-019-01462-5.

[38]. Kalbasi A, June CH, Haas N, Vapiwala N. Radiation and immunotherapy: a synergistic combination. J Clin Invest 2013;123:2756-63. doi:10.1172/JCI69219. [PubMed: 23863633]

[39]. Wild AT, Herman JM, Dholakia AS, Moningi S, Lu Y, Rosati LM, et al. Lymphocyte-Sparing Effect of Stereotactic Body Radiation Therapy in Patients With Unresectable Pancreatic Cancer. Int J Radiat Oncol Biol Phys 2016;94:571-9. doi:10.1016/j.ijrobp.2015.11.026. [PubMed: 26867885]

[40]. Hammi A, Paganetti H, Grassberger C. 4D blood flow model for dose calculation to circulating blood and lymphocytes. Physics in Medicine \& Biology 2020;65:055008. doi:10.1088/1361-6560/ab6c41. [PubMed: 32119649] 
[41]. Zhao Q, Chen G, Ye L, Shi S, Du S, Zeng Z, et al. Treatment-duration is related to changes in peripheral lymphocyte counts during definitive radiotherapy for unresectable stage III NSCLC. Radiat Oncol 2019;14:86. doi:10.1186/s13014-019-1287-z. [PubMed: 31133034]

[42]. Poleszczuk JT, Luddy KA, Prokopiou S, Robertson-Tessi M, Moros EG, Fishman M, et al. Abscopal Benefits of Localized Radiotherapy Depend on Activated T-cell Trafficking and Distribution between Metastatic Lesions. Cancer Res 2016;76:1009-18. doi:10.1158/0008-5472.CAN-15-1423. [PubMed: 26833128]

[43]. Walker R, Poleszczuk J, Pilon-Thomas S, Kim S, Anderson AARA, Czerniecki BJ, et al. Immune interconnectivity of anatomically distant tumors as a potential mediator of systemic responses to local therapy. Sci Rep 2018;8:9474. doi:10.1038/s41598-018-27718-1. [PubMed: 29930290]

[44]. West CML, Davidson SE, Elyan SAG, Valentine H, Roberts SA, Swindell R, et al. Lymphocyte radiosensitivity is a significant prognostic factor for morbidity in carcinoma of the cervix. Int $\mathbf{J}$ Radiat Oncol Biol Phys 2001;51:10-5. [PubMed: 11516845]

[45]. Sanford NN, Pursley J, Noe B, Yeap BY, Goyal L, Clark JW, et al. Protons versus Photons for Unresectable Hepatocellular Carcinoma: Liver Decompensation and Overall Survival. Int J Radiat Oncol Biol Phys 2019;105:64-72. doi:10.1016/j.ijrobp.2019.01.076. [PubMed: 30684667]

[46]. Davuluri R, Jiang W, Fang P, Xu C, Komaki R, Gomez DR, et al. Lymphocyte Nadir and Esophageal Cancer Survival Outcomes After Chemoradiation Therapy. Int J Radiat Oncol Biol Phys 2017;99:128-35. doi:10.1016/j.ijrobp.2017.05.037. [PubMed: 28816138]

[47]. Routman DM, Garant A, Lester SC, Day CN, Harmsen WS, Sanheuza CT, et al. A Comparison of Grade 4 Lymphopenia With Proton Versus Photon Radiation Therapy for Esophageal Cancer. Adv Radiat Oncol 2019;4:63-9. doi:10.1016/j.adro.2018.09.004. [PubMed: 30706012]

[48]. Alfonso JCL, Poleszczuk J, Walker R, Kim S, Pilon-Thomas S, Conejo-Garcia JJ, et al. Immunologic Consequences of Sequencing Cancer Radiotherapy and Surgery. JCO Clin Cancer Inform 2019:1-16. doi:10.1200/CCI.18.00075.

[49]. Eftimie R, Macnamara CK, Dushoff J, Bramson JL, Earn DJD. Bifurcations and Chaotic Dynamics in a Tumour-Immune-Virus System. Math Model Nat Phenom 2016;11:65-85. doi:10.1051/mmnp/201611505.

[50]. Wang L, Simons DL, Lu X, Tu TY, Solomon S, Wang R, et al. Connecting blood and intratumoral $\mathrm{T}$ reg cell activity in predicting future relapse in breast cancer. Nature Immunology 2019 2019;30:1. doi:10.1038/s41590-019-0429-7.

[51]. Spiotto M, Fu Y-X, Weichselbaum RR. The intersection of radiotherapy and immunotherapy: mechanisms and clinical implications. Science Immunology 2016;1:eaag1266-6. doi:10.1126/ sciimmunol.aag1266. [PubMed: 28018989]

[52]. Falcke SE, Rühle PF, Deloch L, Fietkau R, Frey B, Gaipl US. Clinically Relevant Radiation Exposure Differentially Impacts Forms of Cell Death in Human Cells of the Innate and Adaptive Immune System. Ijms 2018;19:3574. doi:10.3390/ijms19113574.

[53]. Anichini A, Tassi E, Grazia G, Mortarini R. The non-small cell lung cancer immune landscape: emerging complexity, prognostic relevance and prospective significance in the context of immunotherapy. Cancer Immunology, Immunotherapy 2018;67:1011-22. doi:10.1007/ s00262-018-2147-7. [PubMed: 29516154] 


\section{Highlights}

A mathematical model of lymphocyte depletion by radiotherapy was developed based on measurements in patients during treatment

The model quantifies the ability of short fractionation regimens to mitigate lymphocyte depletion, but also predicts shorter suppression periods and faster recovery

The model predicts the effect of fractionation, prescription dose and timing on circulating lymphocyte counts, and aids in the design of clinical trials aimed at improving the immunogenicity of RT regimens. 


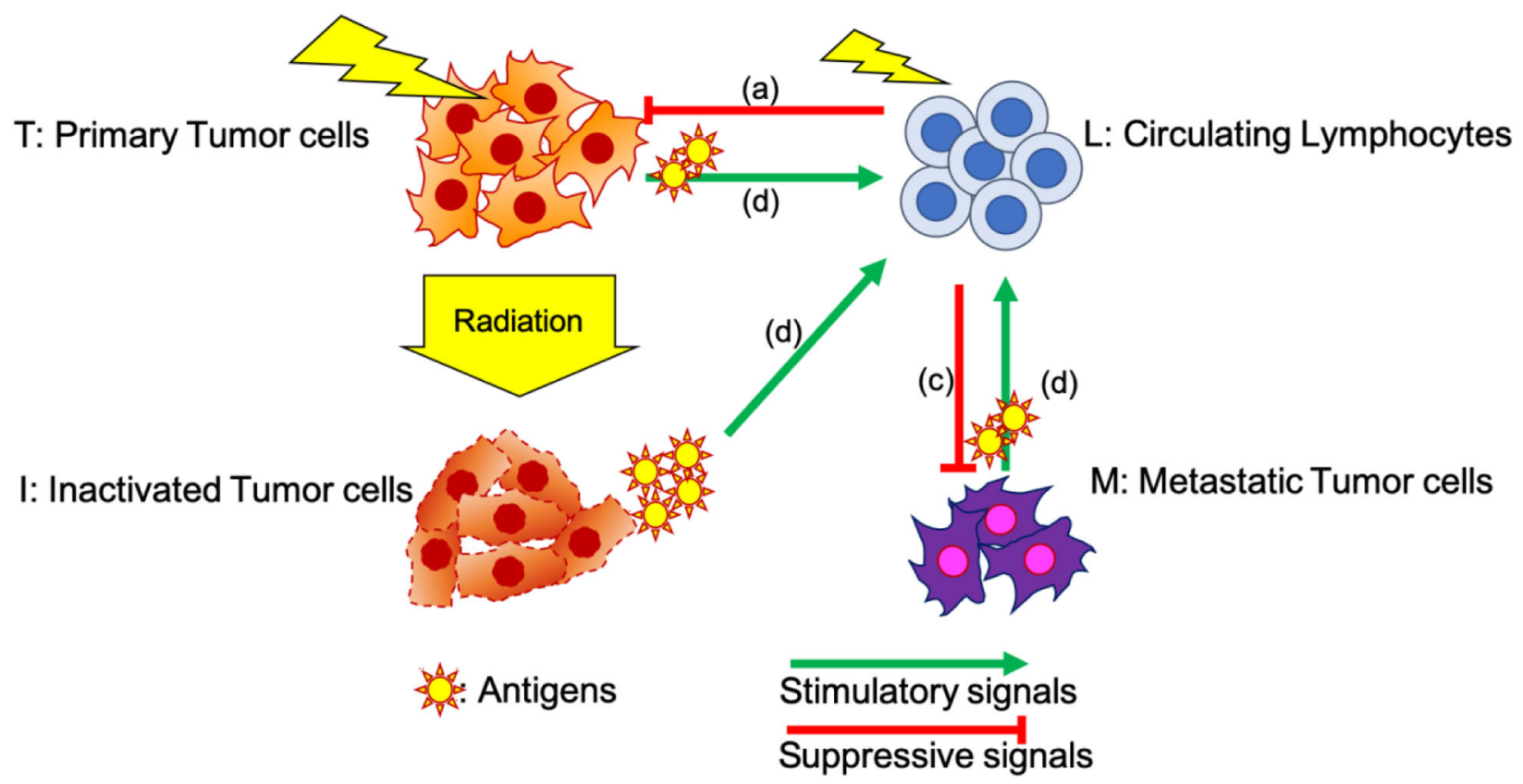

Figure 1.

Schematic illustration of the mechanisms included in the model with arrows indicating their stimulating and inhibiting effects. Letters indicate equations (a) - (d) discussed in the text. Model consists of four compartments: primary tumor cells (T), inactivated tumor cells (I), metastatic tumor cells (M), and circulating lymphocytes (L). Radiation is incident only to primary tumor and circulating lymphocytes. 


\section{Source Data}

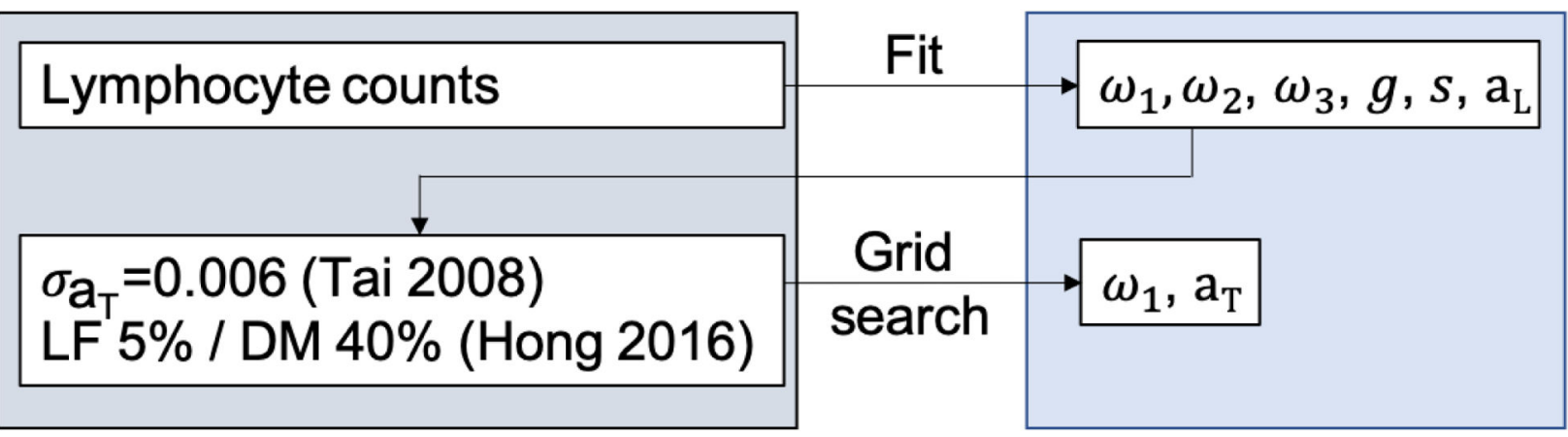

Figure 2.

Process of parameter estimation. Total 6 parameters are fitted to clinical data of circulating lymphocyte counts. Additionally, the immune-induced death rate $\left(\omega_{1}\right)$ and tumor radiosensitivity $\left(a_{T}\right)$ were fitted to acquire $5 \%$ local failure and $40 \%$ distant metastasis rate at two years for a 15-fraction treatment, as observed in our clinical data for this patient cohort. 

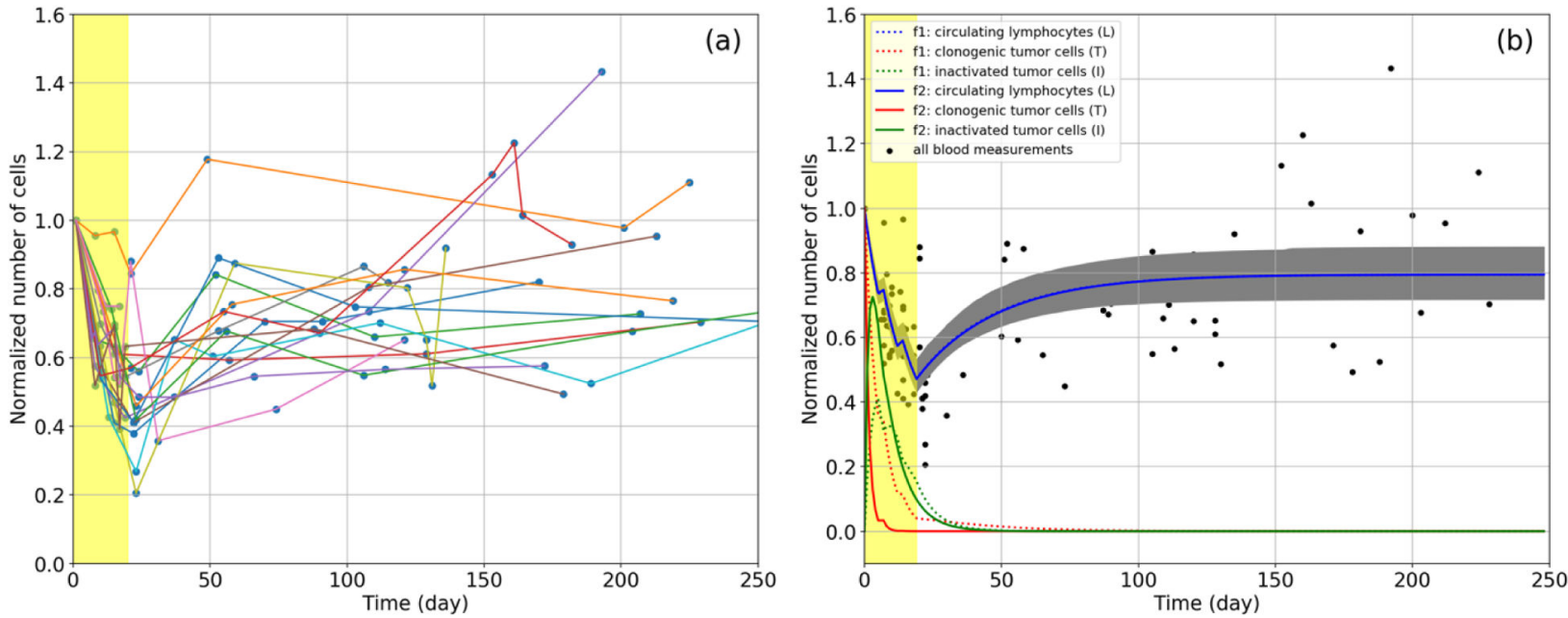

Figure 3.

(a) The number of circulating lymphocytes (CL, clinical blood measurements) and (b) the best fit curves to the model. The number of CLs were normalized to the baseline values. The yellow shaded regions show the radiation treatment periods for 15 fractions. For (a), data points for the same patient are connected. For (b), The grey shaded regions represent the 95\% confidence intervals of fitted curves. Note that the circulating lymphocytes $(\mathrm{L})$ curves of $\mathrm{f} 1$ and $\mathrm{f} 2$ nearly overlapped. $\mathrm{f} 1$ : fitting curves before the grid-search $\mathrm{f} 2$ : fitting curves after the grid-search 

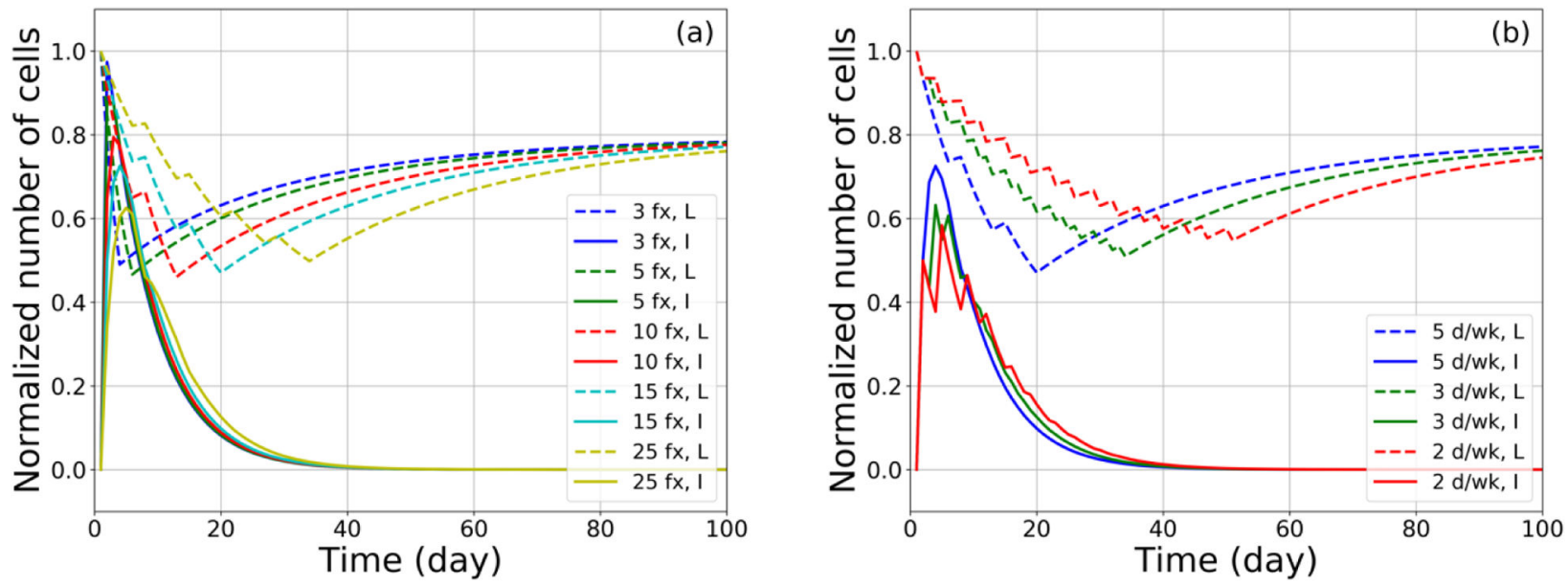

Figure 4.

The simulated trajectories of circulating lymphocytes (L: dotted lines) and inactivated tumor cells (I: solid lines) for (a) different total fractions (fx) with single daily delivery and (b) different treatment days per week (d/wk) with 15 fractions. The parameters used to create these trajectories are the derived population averages given in Table 2, for an analysis how sensitive these simulations are to patient-specific variations in the parameters see our sensitivity analysis in the supplementary materials, section 3 


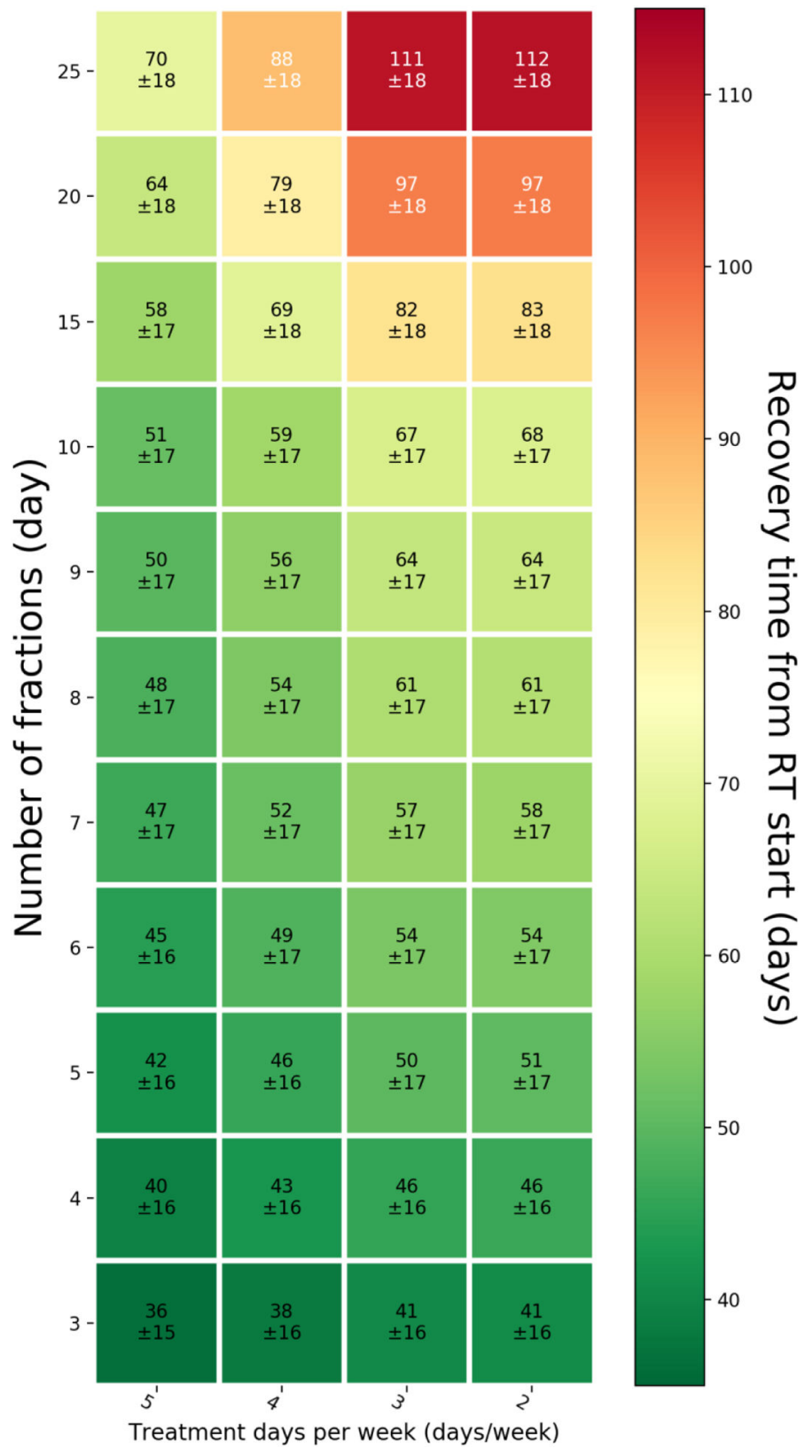

Figure 5.

Lymphocyte depletion behaviors for different number of fractions and treatment days per week. Recovery days from the start of radiotherapy (RT) to the day of circulating lymphocyte recovered back to $70 \%$ of the baseline. The average and standard deviation were obtained with 1,000 parameter sets used for $95 \%$ confidence intervals in Table 1. 


\section{Table 1}

Summary of the parameters included in the fitting procedure. CI: confidence interval

\begin{tabular}{|c|c|c|c|c|c|}
\hline & Parameter & Function & Fitting Constraints & Value $(95 \%$ CI) & References \\
\hline \multirow[t]{4}{*}{ Fixed (4) } & a & Tumor growth & - & $0.01 \mathrm{~d}^{-1}$ & [30] \\
\hline & $\alpha_{T} / \beta_{T}$ & Tumor - LQ cell kill & - & $14.3 \mathrm{~Gy}$ & [34] \\
\hline & $f$ & Lymphocyte decay rate & - & $0.033 \mathrm{~d}^{-1}$ & [33] \\
\hline & $r$ & Inactivated tumor cell decay rate & - & $0.14 \mathrm{~d}^{-1}$ & [32] \\
\hline \multirow[t]{6}{*}{ Fitted (7) } & $\omega_{1}$ & $\begin{array}{l}\text { Tumor-directed lymphocyte } \\
\text { efficiency }\end{array}$ & $10^{-3}-10^{0} \mathrm{~d}^{-1}$ & $0.119 \mathrm{~d}^{-1}(0.089-0.141)$ & {$[26,27]$} \\
\hline & $\begin{array}{l}\omega_{2} \\
\omega_{3}\end{array}$ & $\begin{array}{l}\text { Tumor / Inactivated tumor - } \\
\text { lymphocyte recruitment constant }\end{array}$ & $10^{-3}-10^{0} \mathrm{~d}^{-1}$ & $\begin{array}{l}0.003 \mathrm{~d}^{-1}(0.001-0.0105) \\
0.009 \mathrm{~d}^{-1}(0.001-0.0575)\end{array}$ & {$[26,27]$} \\
\hline & $g$ & Half-saturation constant & $10^{8}-10^{14}$ & $\begin{array}{l}7.330 \times 10^{10}\left(6.130 \times 10^{10}-10.0 \times\right. \\
\left.10^{10}\right)\end{array}$ & {$[26,27]$} \\
\hline & $s$ & Lymphocyte regeneration & $0-5.61 \times 10^{9}$ & $\begin{array}{l}1.470 \times 10^{8} \mathrm{~d}^{-1}\left(1.330 \times 10^{8}-1.620 \times\right. \\
\left.10^{8}\right)\end{array}$ & [37] \\
\hline & $a_{T}$ & Tumor - LQ cell kill & - & $0.139 \mathrm{~Gy}^{-1}(0.095-0.183)$ & [34] \\
\hline & $a_{L}$ & Lymphocytes - LQ cell kill & $10^{-2}-10^{0}$ & $0.737 \mathrm{~Gy}^{-1}(0.513-0.999)$ & {$[20,43]$} \\
\hline
\end{tabular}

